\title{
Convective overshooting in the evolution and seismology of $\eta$ Bootis
}

\author{
M. P. Di Mauro ${ }^{1,2}$, J. Christensen-Dalsgaard ${ }^{2,3}$, H. Kjeldsen ${ }^{2,3}$, T. R. Bedding ${ }^{4}$, and L. Paternò ${ }^{5}$ \\ 1 INAF-Osservatorio Astrofisico di Catania, via S. Sofia 78, 95123 Catania, Italy \\ 2 Teoretisk Astrofysik Center, Danmarks Grundforskningsfond \\ 3 Institut for Fysik og Astronomi, Aarhus Universitet, Bygn. 520, Ny Munkegade, 8000 Aarhus C, Denmark \\ e-mail: jcd@phys.au.dk; hans@phys.au.dk \\ 4 School of Physics A28, University of Sydney, NSW 2006, Australia \\ e-mail: bedding@physics.usyd.edu.au \\ 5 Dipartimento di Fisica e Astronomia dell’Università di Catania, via S. Sofia 78, 95123 Catania, Italy \\ e-mail: lpaterno@ct.astro.it
}

Received 6 May 2002 / Accepted 24 March 2003

\begin{abstract}
In the near future frequencies of solar-like oscillation will be observed by the several planned space missions. In order to assess the information that will be available from such observations, we consider theoretical predictions of the frequency spectrum of oscillations of the star $\eta$ Bootis, which shows solar-type pulsations. We present results obtained by comparing "classical" evolution models with models computed by taking into account overshooting from the convective core.
\end{abstract}

Key words. stars: individual: $\eta$ Bootis - stars: evolution - stars: oscillations

\section{Introduction}

In view of the rapidly increasing prospects for observations of stellar oscillation data, not least thanks to upcoming space missions, we have begun to prepare a list of primary science targets suitable for seismological analysis by investigating the theoretical prediction of the spectra of oscillations of these stars.

The subgiant HR 5235, better known as $\eta$ Bootis, is a well-studied bright star of spectral type G0 IV and a good candidate for asteroseismic studies since theory (Kjeldsen \& Bedding 1995) predicts a relatively large surface amplitude of oscillation.

The first observational success was achieved by Kjeldsen et al. (1995) by using an approach based on the measurements of the variation of the equivalent width of hydrogen Balmer lines. They found in $\eta$ Boo the signature of solar-like pulsations which, similarly to those observed in the Sun, are believed to be excited stochastically by turbulent convection. Unfortunately, direct radial velocity measurements failed to confirm the presence of an excess of power (Brown et al. 1997) to be attributed to solar-like oscillations.

Only recently, Kjeldsen et al. (2003) by observing this star both in the Balmer-line equivalent width and in velocity have confirmed the presence of solar-like oscillations, characterized by a large frequency separation $\Delta v=(40.47 \pm 0.05) \mu \mathrm{Hz}$ and a

Send offprint requests to: M. P. Di Mauro,

e-mail: mdm@ct.astro.it small frequency separation $\delta v=(3.06 \pm 0.14) \mu \mathrm{Hz}$. They identified $21 \mathrm{p}$-mode frequencies in the range $600-1100 \mu \mathrm{Hz}$, with harmonic degrees $l=0-2$. Also, Carrier et al. (2003) very recently reported detection of oscillations, with properties essentially consistent with those obtained in previous analyses, from Doppler-velocity observations. Although these results need to be coordinated, for the purpose of this study of the seismic behaviour of $\eta$ Boo we may assume that the star displays solartype oscillations.

It has already been demonstrated that "classical" evolution models can account for most of the observed properties of $\eta$ Boo (Christensen-Dalsgaard et al. 1995a; Guenther \& Demarque 1996). However, it is likely (Shaviv \& Salpeter 1973) that overshoot from the convective core, ignored in these early studies, may be significant during the main-sequence phase, leading to important consequences in the successive evolutionary phase of helium burning. In fact, the standard description of convection does not consider that material motions and mixing might extend beyond the boundary defined by the Schwarzschild criterion.

Thus, it may be of interest to investigate the effects of such an overshoot on the evolution and oscillation properties of $\eta$ Boo. We will show that also the models which include convective overshooting can reasonably reproduce the preliminary observed spectrum (Kjeldsen et al. 2003), and that there is no evidence for excluding mixing outside the convectively unstable regions. 


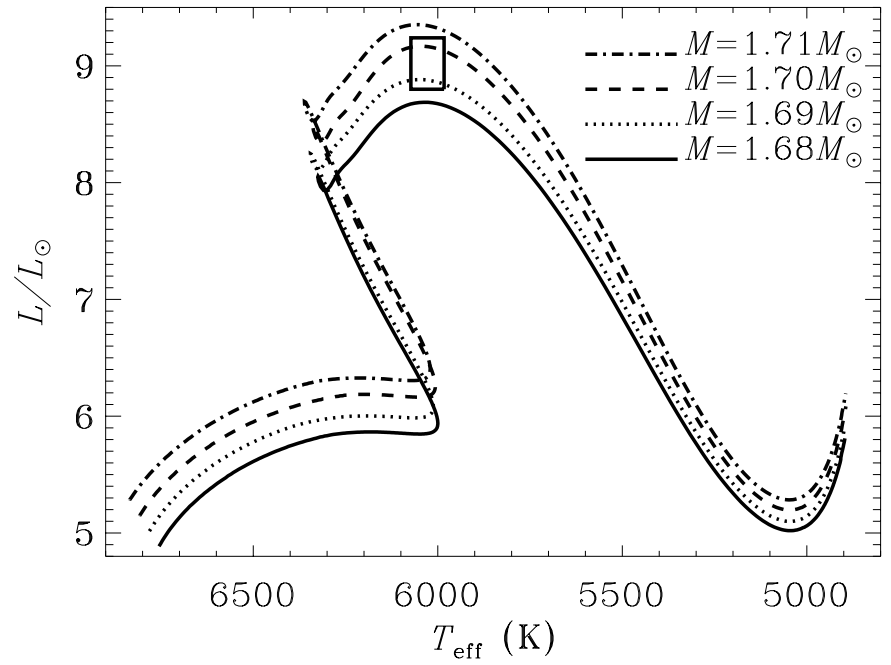

Fig. 1. Resulting evolution tracks plotted in an $\mathrm{H}-\mathrm{R}$ diagram for several masses, assuming the observed metallicity $(Z=0.04)$. The rectangle defines the one-sigma error box for the observed luminosity and effective temperature.

It is clear that only more accurate observations will allow investigation of the subtle properties of the interior of this star and discrimination among the several possible evolutionary scenarios which seem consistent with the existing spectroscopic and asteroseismic observations. For this reason, $\eta$ Boo has been selected as primary target for a number of planned space missions, firstly MOST (Microvariability \& Oscillations of STars; Matthews 1998), to be launched in 2003, and subsequently MONS (Measuring Oscillations in Nearby Stars; Christensen-Dalsgaard 2002) planned to fly aboard the Rømer satellite, which will permit to go beyond the current detection thresholds of Earth-based instruments to reveal finer detail of the $\mathrm{p}$-mode eigenspectrum of this star.

\section{Models}

We produced a grid of models of $\eta$ Boo evolved from chemically uniform models on the zero-age main sequence (ZAMS), in approximately 400 time steps, by using the evolution code of Christensen-Dalsgaard (1982). All the models presented here are calculated with the EFF equation of state (Eggleton et al. 1973), OPAL opacities (Iglesias \& Rogers 1996), Bahcall \& Pinsonneault (1995) nuclear cross sections and the mixinglength formalism (MLT) for convection.

Special care has been taken in the choice of the observed stellar parameters among the several values quoted in literature. We adopted the luminosity $L / L_{\odot}=9.02 \pm 0.22$ obtained by Bedding et al. (1997) and Mallik (1999) based on the Hipparcos parallax $\pi=(88.17 \pm 0.75)$ mas. We assumed an effective temperature $T_{\text {eff }}=(6028 \pm 45) \mathrm{K}$, obtained as a weighted average of the values reported by Bell \& Gustafsson (1989), Blackwell \& Lynas-Gray (1994), Flower (1996), Lèbre et al. (1999) and Mallik (1999). A crucial quantity is the iron abundance, whose logarithmic value relative to the solar one has been taken to be $[\mathrm{Fe} / \mathrm{H}]=(0.305 \pm 0.051)$, as determined by Taylor (1996), who regards this star as a super-metal-rich

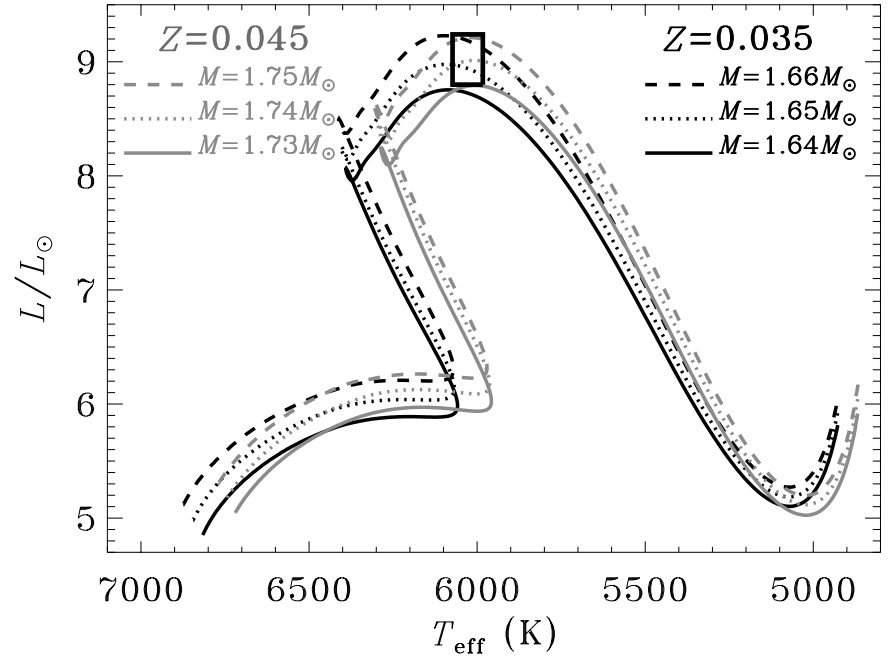

Fig. 2. Evolutionary tracks in the H-R diagram calculated for several masses by varying the metallicity in the interval of errors to match the observed values of $T_{\text {eff }}$ and $L$. The grey tracks are obtained for $Z=0.045$, while the black tracks are obtained for $Z=0.035$.

star. The hydrogen abundance has been assumed to be $X=0.7$, so that $Z=0.04 \pm 0.005$.

The resulting evolutionary tracks, plotted in an $\mathrm{H}-\mathrm{R}$ diagram (Fig. 1), are calculated for several masses using a mixinglength parameter $(\alpha=1.8837)$ calibrated on a solar model constructed with the same microphysics as the stellar models. Given the input parameters, we produced a family of possible models which are characterized by the variables which are not determined by observations, namely the mass $M$ and the age of the star.

In order to investigate the sensitivity of the models to the assumed parameters, we have computed additional sets of evolutionary sequences by varying the metallicity in the uncertainty range, while all other parameters were left unchanged. Evolutionary tracks computed using $Z=0.035$ and $Z=0.045$ are shown in Fig. 2. The results indicate that the decrease in metallicity requires a decrease in mass and vice versa in order to match the observed location in the $\mathrm{H}-\mathrm{R}$ diagram. The uncertainty in the observed value of $Z$ introduces an uncertainty in the determination of the mass of $\eta$ Boo whose value, assuming the above parameters, seems to be limited to the range $M=(1.64-1.75) M_{\odot}$.

We are also able to confirm (Christensen-Dalsgaard et al. 1995a) that variations on the assumed mixing-length parameter produce small changes in luminosity and effective temperature of the models in the post-main-sequence stage.

To test the effects of overshooting, we also computed a family of evolutionary sequences by including overshoot from the convective core during the main-sequence phase and studying the oscillations of these models. The overshoot produces a chemical and thermal mixing in the region where the turbulent motions penetrate, from the edge of the convective core to a radial extent $\ell_{\mathrm{ov}}$ defined by a parameter $\alpha_{\mathrm{ov}}$ :

$\ell_{\mathrm{ov}}=\alpha_{\mathrm{ov}} \min \left(r_{\mathrm{c}}, H_{\mathrm{p}}\right)$, 


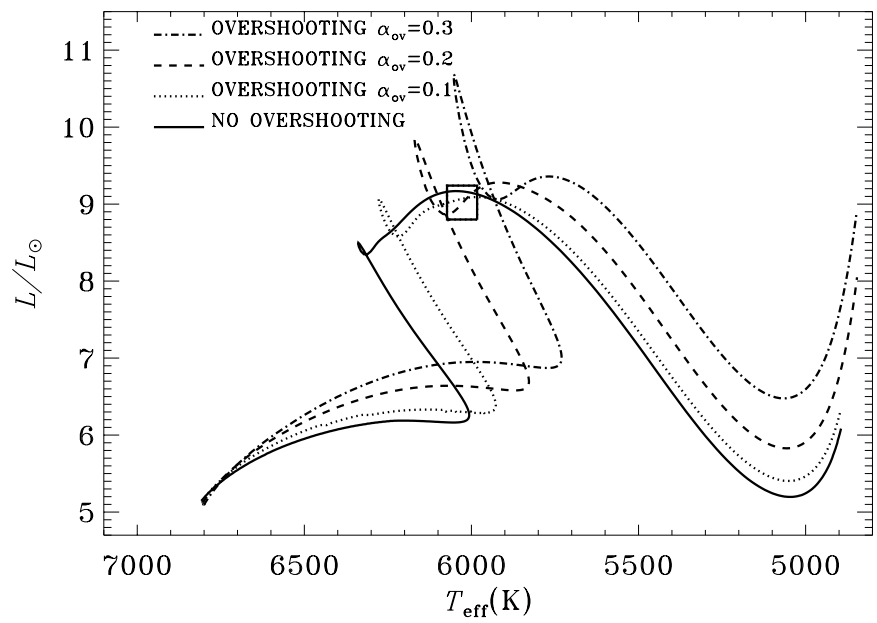

Fig. 3. The effect of the overshooting on the evolution of $\eta$ Boo in the $\mathrm{H}-\mathrm{R}$ diagram, calculated for increasing values of the extent of the overshooting from the the convective stellar core. Here models are calculated with $M=1.7 M_{\odot}$ and $Z=0.04$.

where $H_{\mathrm{p}}$ is the pressure scale height at the edge of the core, $r_{\mathrm{c}}$ is the radius of the convective core and $\alpha_{\mathrm{ov}}$ is a nondimensional parameter typically below unity. The expression (1) is used to limit the extent of the overshoot region to be no larger than a fraction $\alpha_{\mathrm{ov}}$ of the size of the core. In fact, stellar models with small convective cores might have very high values of $H_{\mathrm{p}}$ and in this case $r_{\mathrm{c}}$ is used.

Here, models have been computed with increasing overshoot distance, by varying $\alpha_{\text {ov }}$ from 0.1 to 0.3 . The resulting evolutionary tracks are shown in Fig. 3, compared with the track computed without overshooting. All sequences use the same mass and heavy-element abundance; interestingly, for $\alpha_{\mathrm{ov}} \leq 0.2$ this provides a match to the observed $\left(T_{\mathrm{eff}}, L\right)$ of $\eta$ Boo, the tracks crossing at this location in the diagram. On the other hand, we conclude that models with larger overshooting, $\alpha_{\text {ov }}>0.25$, are not consistent with the observed values of luminosity and effective temperature; this appears to be the case regardless of the choice of mass and heavy-element abundance. The ZAMS models are very similar independently of overshooting, since they are chemically uniform.

The location of the star in the Hertzsprung-Russell diagram identifies $\eta$ Boo as being in the post-main-sequence phase of evolution. It has a helium core, having exhausted its central hydrogen, and it is in the shell-hydrogen-burning phase, evolving toward the red giant branch. The models have a convective envelope extending from the base located at about $r_{\mathrm{cb}} \simeq 0.84 R$ into the photosphere; in particular, the near-surface convection is sufficiently strong to excite stochastically the modes to the observed amplitudes (e.g. Houdek et al. 1999).

Overshooting results in an extension of the mixed core, and hence makes more hydrogen fuel available for nuclear burning during the evolution. At fixed effective temperature, a model with overshooting is slightly less luminous and less evolved than a model without overshooting, during core hydrogen burning; the main-sequence lifetime is increased by about $15 \%$ for $\alpha_{\mathrm{ov}}=0.2$.
Table 1. Age, luminosity $L$ (in units of the solar luminosity $L_{\odot}$ ), effective temperature $T_{\text {eff }}$, surface radius $R$ and distance $r_{\mathrm{cb}}$ of the base of the convective envelope from the centre in units of $R$, for standard models of $\eta$ Bootis (no overshooting), computed with $M=1.7 M_{\odot}$ and $Z=0.04$.

\begin{tabular}{cccccc}
\hline \hline & Age (Gy) & $L / L_{\odot}$ & $T_{\text {eff }}(\mathrm{K})$ & $R / R_{\odot}$ & $r_{\mathrm{cb}} / R$ \\
\hline 1 & 2.367 & 9.159 & 6074 & 2.74 & 0.851 \\
2 & 2.371 & 9.166 & 6062 & 2.75 & 0.848 \\
3 & 2.375 & 9.169 & 6050 & 2.76 & 0.845 \\
4 & 2.378 & 9.168 & 6038 & 2.77 & 0.842 \\
5 & 2.382 & 9.164 & 6025 & 2.78 & 0.839 \\
6 & 2.385 & 9.157 & 6013 & 2.79 & 0.836 \\
7 & 2.388 & 9.147 & 6001 & 2.80 & 0.833 \\
8 & 2.390 & 9.134 & 5988 & 2.81 & 0.829 \\
\hline
\end{tabular}

Table 2. Same as Table 1, but including convective overshooting from the core $\left(\alpha_{\mathrm{ov}}=0.1\right)$.

\begin{tabular}{cccccc}
\hline \hline & Age (Gy) & $L / L_{\odot}$ & $T_{\text {eff }}(\mathrm{K})$ & $R / R_{\odot}$ & $r_{\text {cb }} / R$ \\
\hline 1 & 2.395 & 9.02 & 6068 & 2.72 & 0.847 \\
2 & 2.399 & 9.03 & 6058 & 2.73 & 0.845 \\
3 & 2.406 & 9.06 & 6037 & 2.76 & 0.840 \\
4 & 2.410 & 9.07 & 6026 & 2.77 & 0.837 \\
5 & 2.414 & 9.08 & 6016 & 2.78 & 0.834 \\
6 & 2.417 & 9.09 & 6005 & 2.79 & 0.832 \\
7 & 2.420 & 9.09 & 5994 & 2.80 & 0.829 \\
8 & 2.424 & 9.09 & 5982 & 2.81 & 0.826 \\
\hline
\end{tabular}

Table 3. Same as Table 1, but including convective overshooting from the core $\left(\alpha_{\mathrm{ov}}=0.2\right)$.

\begin{tabular}{cccccc}
\hline \hline & Age $(\mathrm{Gy})$ & $L / L_{\odot}$ & $T_{\text {eff }}(\mathrm{K})$ & $R / R_{\odot}$ & $r_{\mathrm{cb}} / R$ \\
\hline 1 & 2.7042 & 8.91 & 6065 & 2.71 & 0.846 \\
2 & 2.7043 & 8.94 & 6055 & 2.72 & 0.844 \\
3 & 2.7044 & 8.98 & 6047 & 2.74 & 0.842 \\
4 & 2.7046 & 9.01 & 6039 & 2.75 & 0.840 \\
5 & 2.7047 & 9.05 & 6031 & 2.76 & 0.838 \\
6 & 2.7049 & 9.10 & 6023 & 2.77 & 0.836 \\
7 & 2.7050 & 9.13 & 6015 & 2.79 & 0.834 \\
8 & 2.7052 & 9.16 & 6007 & 2.80 & 0.832 \\
9 & 2.7054 & 9.19 & 5999 & 2.81 & 0.831 \\
10 & 2.7056 & 9.22 & 5991 & 2.82 & 0.829 \\
\hline
\end{tabular}

The characteristics of models with $M=1.7 M_{\odot}$ and $Z=0.04$ that satisfy the observed constraints on $L$ and $T_{\text {eff }}$ are given in Tables 1-3 with no overshooting, with $\alpha_{\text {ov }}=0.1$ and with $\alpha_{\mathrm{ov}}=0.2$, respectively. According to the stellar evolution constraints, the observational inputs, and with the use of all the possible values of mass and metallicity, our computations show that the age of $\eta$ Boo is about 2.3-2.4 Gyr without overshooting, and between $2.4-2.7 \mathrm{Gyr}$ in presence of overshooting. Note that for $\alpha_{\mathrm{ov}}=0.2$ the relevant models are in 


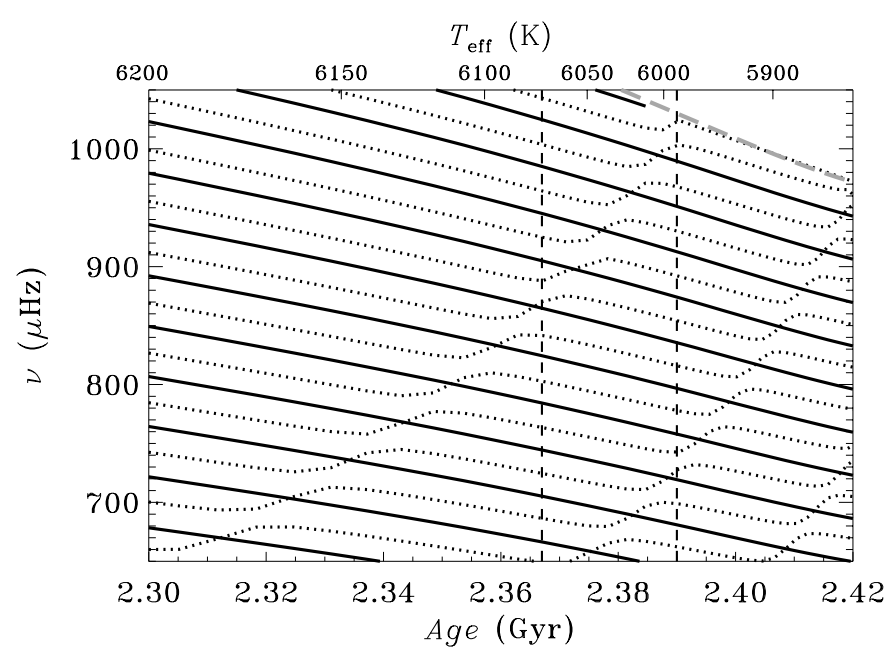

Fig. 4. Evolution of adiabatic frequencies with age and with effective temperature of a model computed without overshooting, with a mass $M=1.7 M_{\odot}$ and $Z=0.04$. The solid lines correspond to modes of degree $l=0$, and the dotted lines to modes with $l=1$. The frequency range corresponds roughly to the range of observed frequencies. The heavy grey dashed line marks the approximate location of the acoustical cut-off frequency at the outer edge of the model. The vertical dashed lines indicate the $1-\sigma$ range in observed $T_{\text {eff }}$.

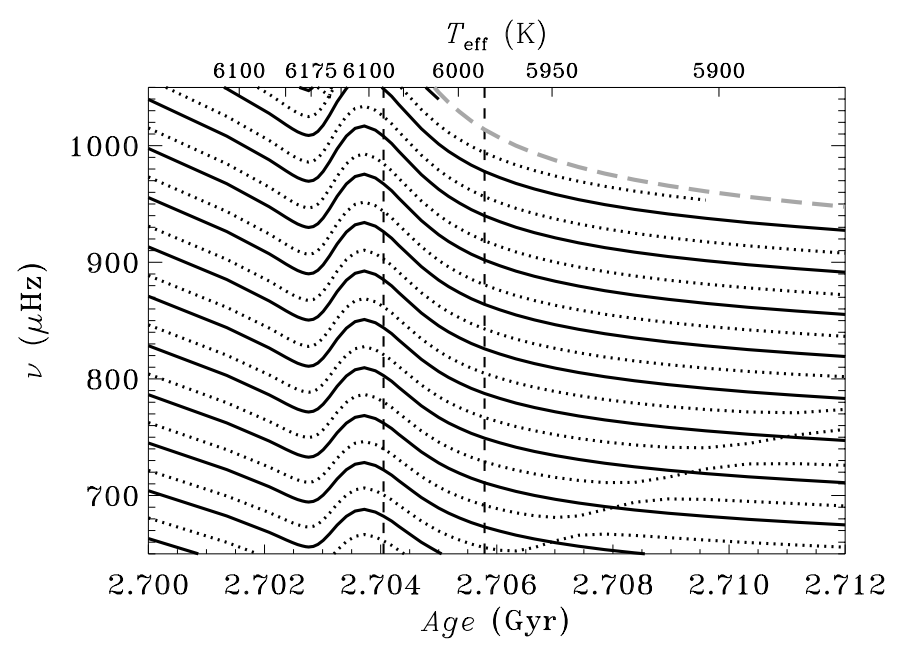

Fig. 5. Same as Fig. 4, but with convective overshooting $\left(\alpha_{\mathrm{ov}}=0.2\right)$. The vertical dashed lines indicate the $1-\sigma$ range in observed $T_{\text {eff }}$. Note that in this case the upper scale in $T_{\text {eff }}$ is non-monotonic, corresponding to the evolutionary stage of the star (see also Fig. 3).

the phase just after the hydrogen shell source has been established, where $T_{\text {eff }}$ is changing considerably more rapidly than in slightly later phases; thus the duration of the passage through the observed range in $T_{\text {eff }}$ is less than one tenth of the duration for $\alpha_{\mathrm{ov}}=0$ and 0.1 .

\section{Pulsation analysis}

\subsection{Prediction of the oscillation frequencies}

We used the adiabatic oscillation code of Christensen-Dalsgaard (Christensen-Dalsgaard \& Berthomieu 1991) to calculate the p-mode eigenfrequencies with harmonic degree $l=0,1,2,3$.

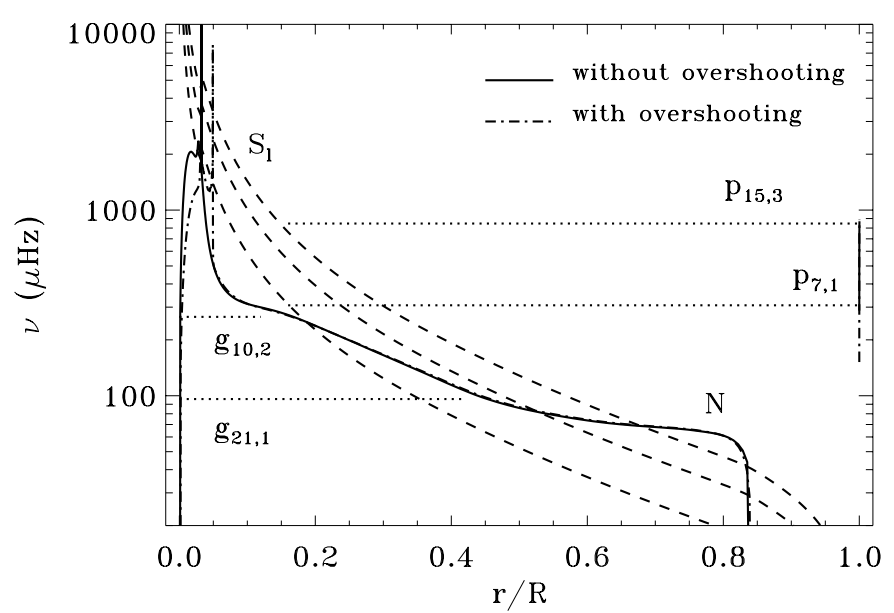

Fig. 6. Propagation diagram for Model 3 without overshooting described in Table 1 and Model 3 with overshooting described in Table 3. The solid line represents the buoyancy frequency $N$ for the model without overshooting, the dot-dashed line represents the buoyancy frequency for the model with overshooting. The dashed lines represent the Lamb frequencies $S_{l}$, increasing with increasing $l$, calculated for $l=1,2,3 . S_{l}$ is practically the same for the two models. The horizontal lines show the regions of propagation of some acoustic modes $\left(\mathrm{p}_{n, l}\right)$ and gravity modes $\left(\mathrm{g}_{n, l}\right)$ of harmonic degree $l$ and radial order $n$.

An easy interpretation of the theoretical and the observational results of our seismic analysis may be obtained by considering the asymptotic properties of the oscillation modes. Theory predicts that oscillation frequencies $v(n, l)$ of acoustic modes, characterized by radial order $n$, at low harmonic degree $l$ should satisfy the asymptotic approximation (Vandakurov 1967; Tassoul 1980):

$v(n, l)=\Delta v\left(n+\frac{l}{2}+\alpha+\frac{1}{4}\right)+\epsilon_{n, l}$,

where $\alpha$ is a function of the frequency and $\epsilon_{n, l}$ is a small correction term that depends on the conditions in the stellar core. $\Delta v$ is the inverse of the sound travel time across the stellar diameter and, according to Eq. (2), it represents approximately the large frequency separation between $p$ modes of same degree and adjacent $n$ :

$\Delta v \simeq v(n, l)-v(n-1, l)$.

$\Delta v$, and hence the general spectrum of acoustic modes, scales approximately as the square root of the mean density, that is, for fixed mass, as $R^{-3 / 2}$.

The oscillation spectrum is also characterized by another parameter, known as the small frequency separation, sensitive to the chemical composition gradient in central regions of the star and hence to its evolutionary state:

$\delta v_{l} \simeq v(n, l)-v(n-1, l+2)$.

The determination of both large and small frequency separation, $\Delta v$ and $\delta v_{l}$, provides a measure of the mass and of the age of the star (e.g. Christensen-Dalsgaard 1988).

Figures 4 and 5 show the evolution of frequencies computed for two evolutionary models calculated with $M=$ $1.7 M_{\odot}, Z=0.04$, without and with overshooting $\left(\alpha_{\mathrm{ov}}=0.2\right)$ 

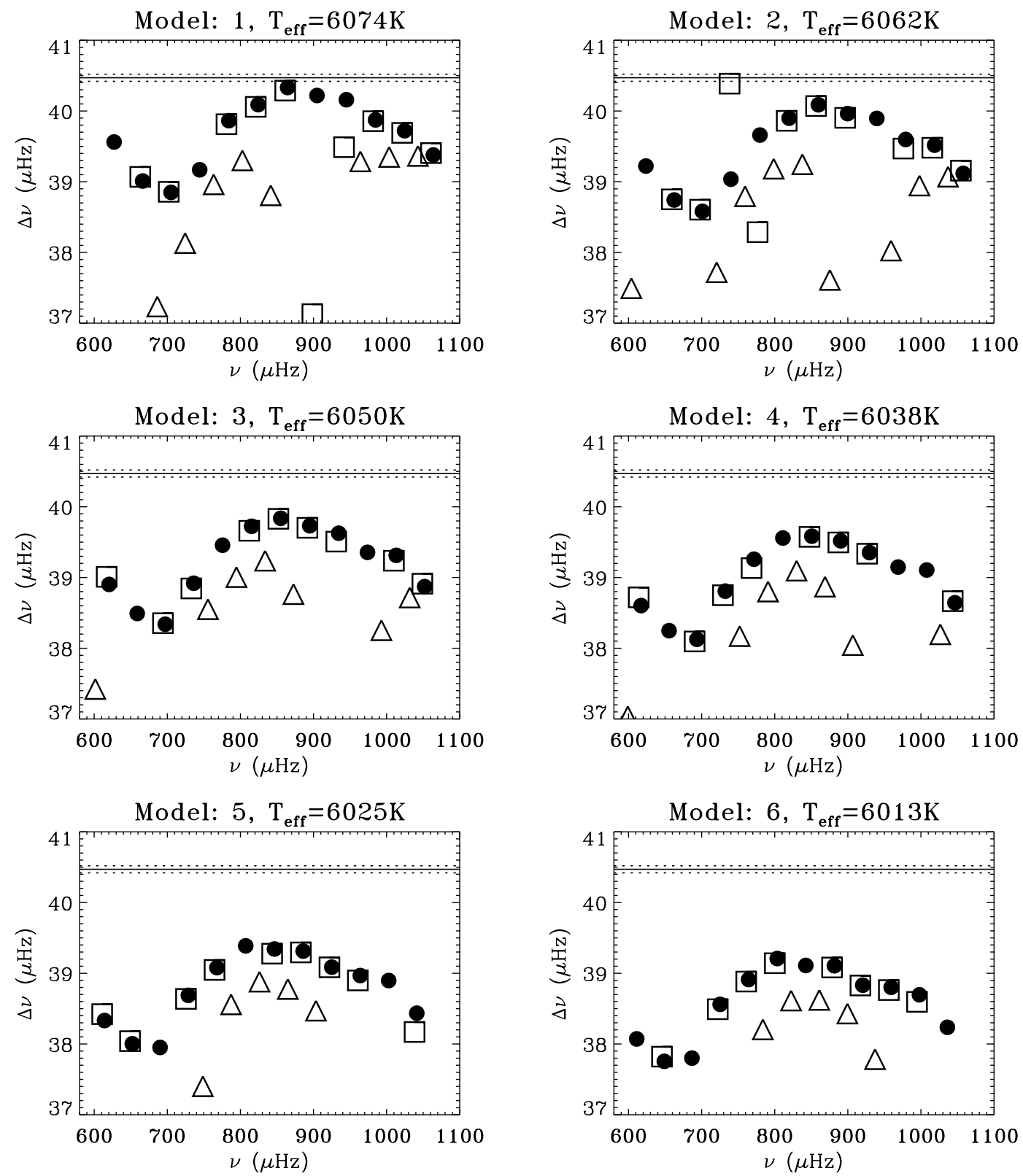

Fig. 7. Large separation as function of the frequency calculated for several models which belong to the same evolution track calculated without overshooting, $M=1.7 M_{\odot}$ and $Z=0.04$. Modes of degree $l=0,1$ and 2 are indicated by filled circles, open triangles and open squares, respectively. The solid line indicates the observed value $\Delta v=40.47 \mu \mathrm{Hz}$ (Kjeldsen et al. 2003) and the dotted lines indicate the observed error limits.

respectively. The range in frequency has been chosen to correspond approximately to the observed range. Also, the ranges in age obtained from the observational error box in the HR diagrams (cf. Fig. 3) are shown by the vertical dashed lines. The location of the acoustical cut-off frequency, decreasing with increasing age, at the top of the atmosphere in the model has been indicated; no standing waves are expected above this frequency, accounting for the lack of modes in the upper right-hand corner of the diagrams. It is interesting that the observed frequencies extend essentially to the acoustical cut-off frequency, within the relevant range of effective temperature.

According to Eq. (2), each plot should be characterized by frequencies which decrease as the star evolves and the radius increases and are almost uniformly spaced by $\Delta v$ at each stage of evolution. Figure 4, for a model without overshooting, shows that, while the radial modes seem to follow closely Eq. (2), the frequencies of some modes with $l=1$ appear to increase and the large separation becomes smaller at certain stages of evolution. This, as already demonstrated (Christensen-Dalsgaard et al. 1995a; Guenther \& Demarque 1996), is a consequence of the increasing frequencies of the g modes associated with the maximum in the buoyancy frequency $N$ in the deep interior of the star (see also Fig. 6). As their frequencies increase with evolution, the g modes undergo avoided crossings with $\mathrm{p}$ modes of the same harmonic degree, giving rise to modes with mixed character.

In contrast, Fig. 5, obtained for a model which includes overshooting from the convective core with $\alpha_{\mathrm{ov}}=0.2$, shows 

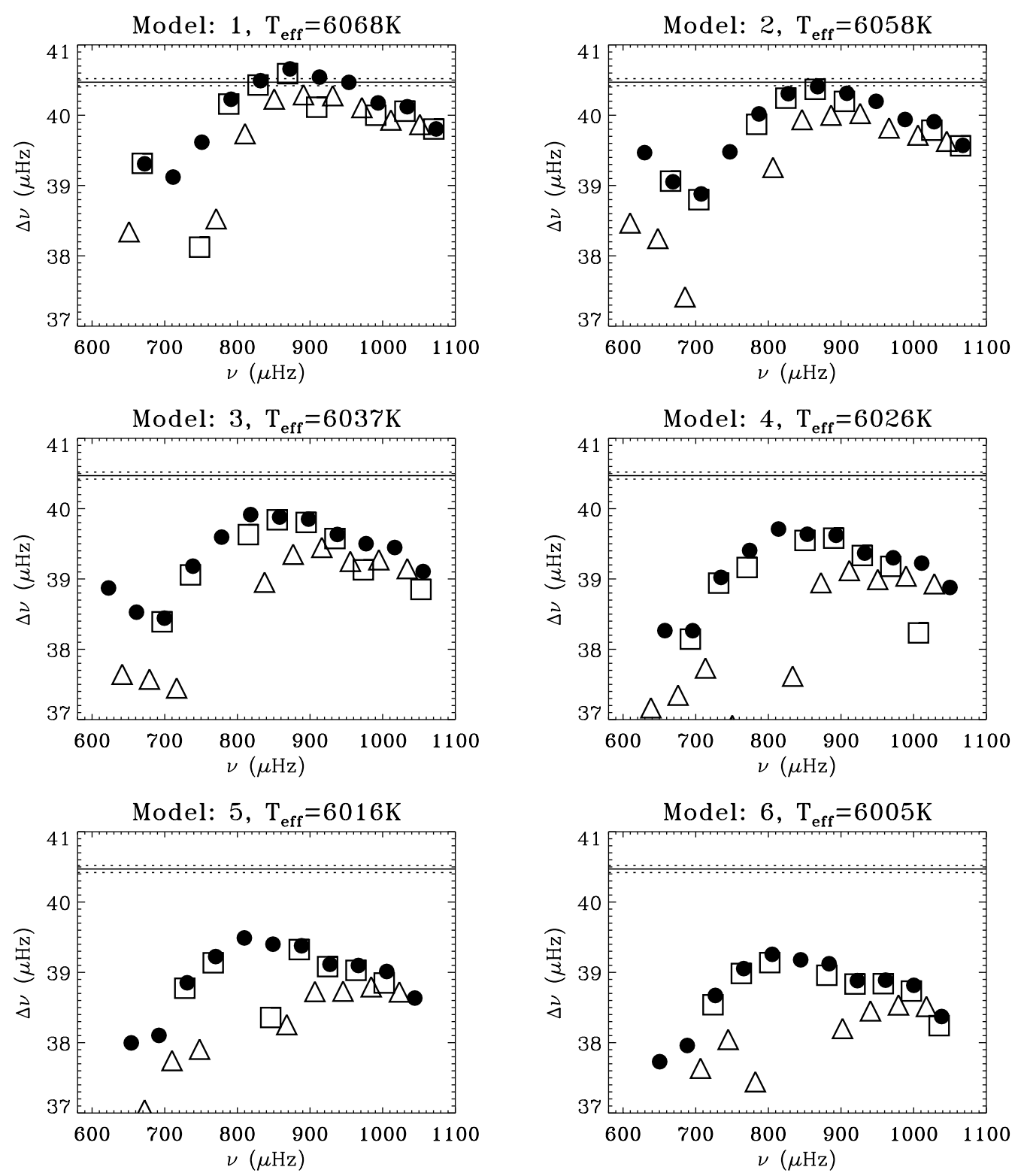

Fig. 8. Large separation as function of the frequency calculated for several models which belong to the same evolution track calculated with overshooting $\left(\alpha_{\mathrm{ov}}=0.1\right), M=1.7 M_{\odot}$ and $Z=0.04$. See caption to Fig. 7 .

that the frequencies are quite regularly spaced; here avoided crossings occur only at low frequencies and for stages of evolution far from the present observed location of this star in the $\mathrm{H}-\mathrm{R}$ diagram.

The two different behaviours can be understood by considering the propagation diagram in Fig. 6 obtained for the Model 3 of Table 1 and Model 3 of Table 3. At nearly the same value of effective temperature and luminosity, the model without overshooting is more evolved than the model with overshooting and its core more contracted, consequently the maximum in its buoyancy frequency is higher and g modes with higher frequencies are allowed to propagate. Thus avoided crossings are more likely in a model without overshooting than in a model with overshooting. We can also predict that the effect of coupling becomes much weaker for modes with $l \geq 2$, since in these cases the gravity waves are trapped in a region well separated from the region of propagation of acoustic waves.

The frequencies in Fig. 5 show a general increase with age just before the model reaches the inferred range in age. This corresponds to the phase of general contraction and increasing effective temperature, at the exhaustion of hydrogen in the convective core: as noticed already in Fig. 3 the relevant models with $\alpha_{\mathrm{ov}}=0.2$ are situated just after this phase. This evolution of the model is also reflected in the upper non-monotonic scale in $T_{\text {eff }}$ in Fig. 5. It is evident from Fig. 3 that the observed location in luminosity identifies the star with the phase just after core hydrogen exhaustion rather than the phase just before it.

\subsection{Comparison with observations}

As an initial comparison with the observed frequencies, Figs. 7-9 show the behaviour of the large separation $\Delta v$ as a 

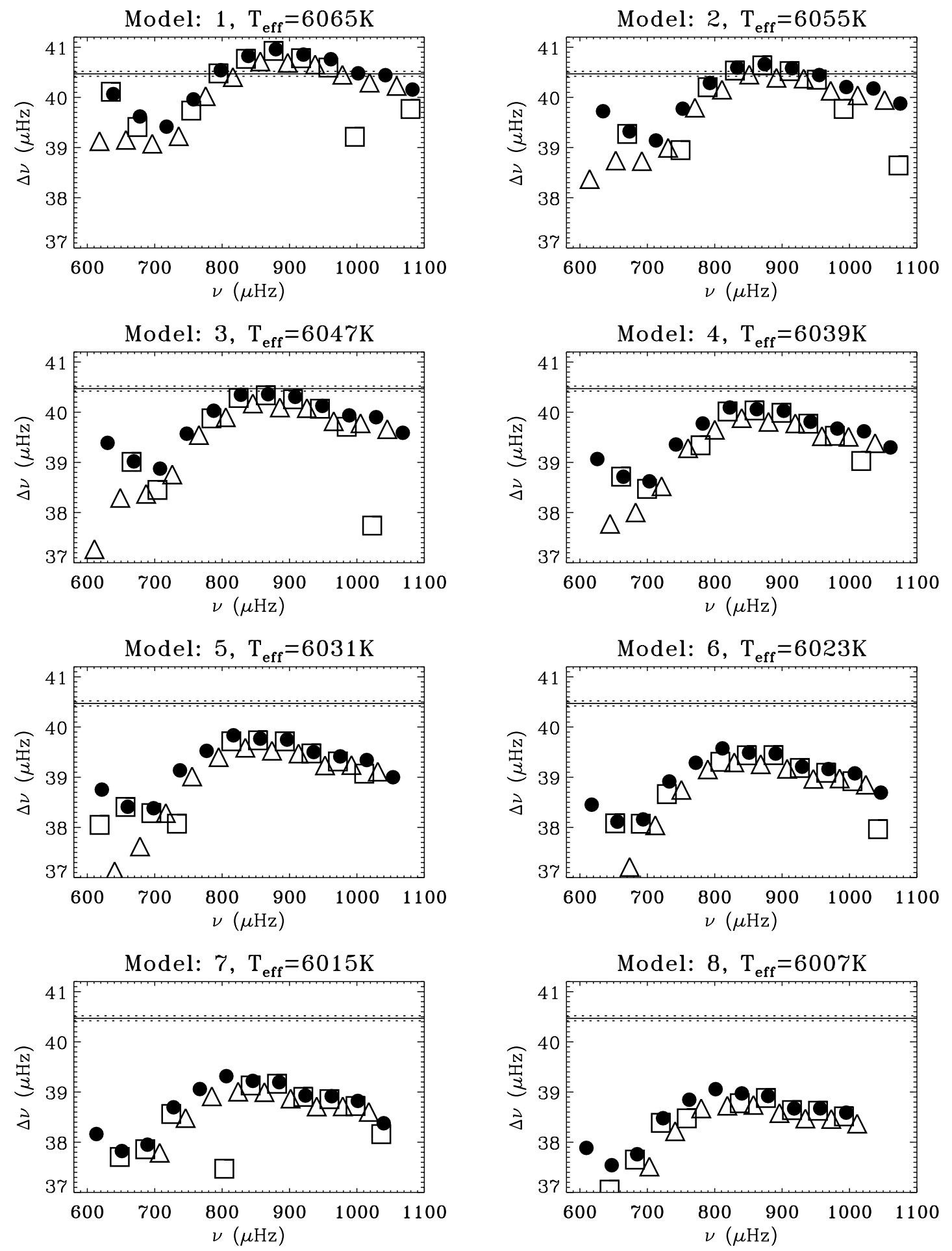

Fig. 9. Large separation as function of the frequency calculated for several models which belong to the same evolution track calculated with overshooting $\left(\alpha_{\mathrm{ov}}=0.2\right), M=1.7 M_{\odot}$ and $Z=0.04$. See caption to Fig. 7 .

function of the frequency, computed for the models described in Tables 1-3. The observed average large separation, $\Delta v=$ $(40.47 \pm 0.05) \mu \mathrm{Hz}$, is indicated by the horizontal line; this was obtained by Kjeldsen et al. (2003) by fitting to the leading term in Eq. (2) separately frequencies for each degree, restricted to the range (600-1000) $\mu \mathrm{Hz}$, and averaging the results. The large separation of the models decreases as the star evolves towards lower effective temperature and larger radius. As it has also been found in the solar case, the modelled $\Delta v$ shows a substantial dependence on frequency. Also, the nonradial modes, with $l=1$ and 2 , show considerably more scatter than do the radial modes, particularly for the models with no or little overshooting (Figs. 7 and 8). This is associated with the avoided crossings (cf. Fig. 4) which introduce a less regular structure in the frequency spectrum. Even so, from all the possible calculations it can be concluded that models can be found in the observed 

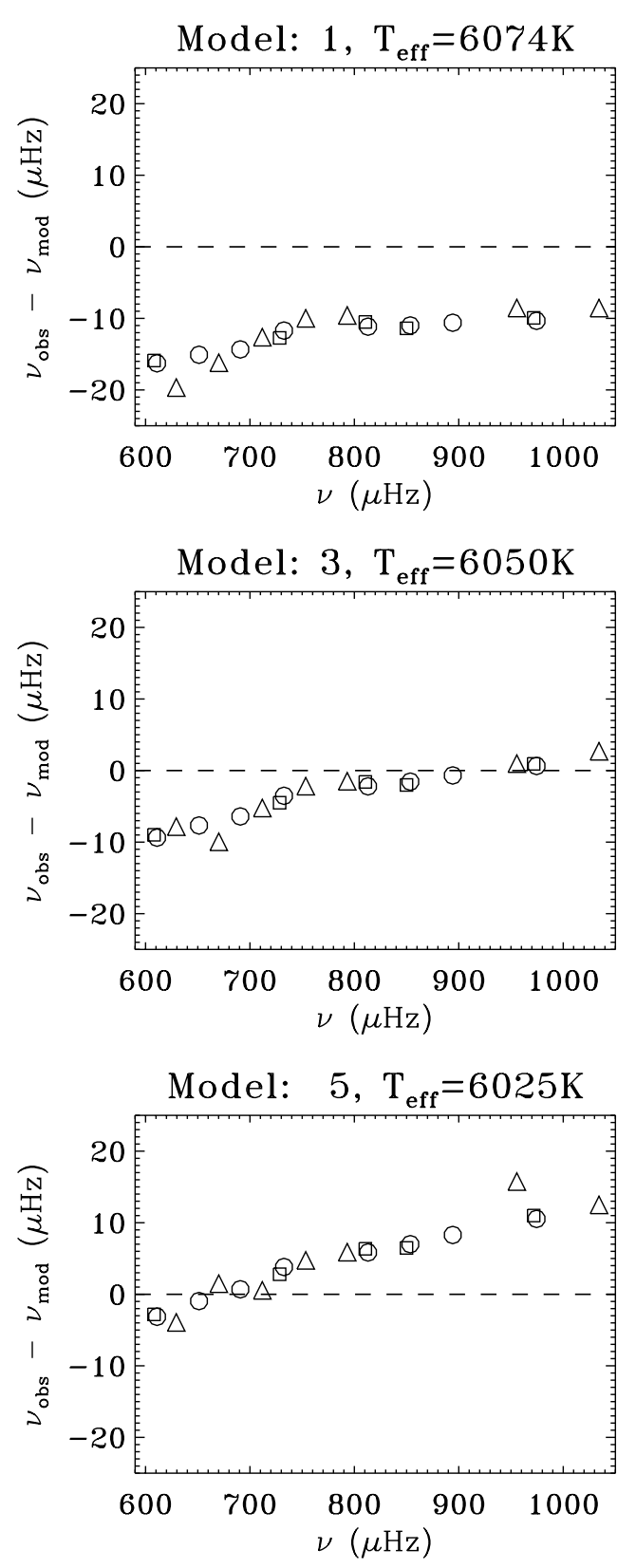
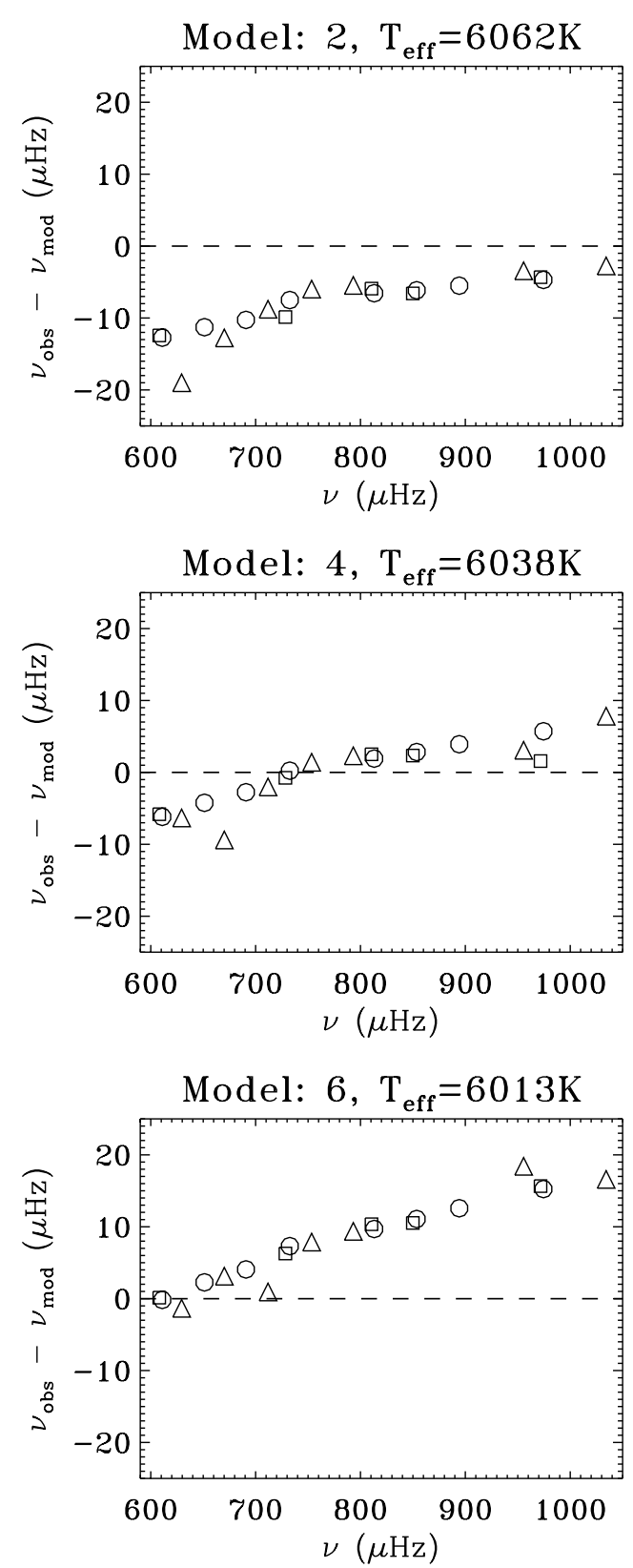

Fig. 10. Differences between observed and theoretical frequencies as function of the frequency calculated for several models which belong to the same evolution track calculated without including overshooting from the convective core and described in Table 1. Open circles represent modes with $l=0$, open triangles represent modes with $l=1$, while open squares represent modes with $l=2$.

range of effective temperature which reproduce the observed large separation, in all three cases of varying overshooting.

A more detailed comparison between the theoretical oscillation spectra and the observed data is provided in Figs. 10-12, which show the differences between the observed and the theoretical frequencies calculated for models without and with overshooting, with characteristics listed in Tables 1-3. A difficulty arises with the identification of the mode orders in the comparison between the observed and computed frequencies. For the observed frequencies the order can be estimated by assuming that the frequencies approximately satisfy the asymptotic relation (2); this evidently refers only to those modes that behave predominantly like $\mathrm{p}$ modes. On the other hand, the identification of the radial order assigned by the pulsation code, according to the Scuflaire (1974) definition, is mathematically well defined, in the sense that the order does not change along the curves shown, e.g., in Fig. 4, but does not take into account the physical nature of the modes. Thus the theoretical order for a given p-mode branch increases by one when the mode passes through an avoided crossing. For any given model this is reflected by the presence in the frequency spectrum of modes with predominantly g-mode character. As a pragmatic approach, we have carried out the comparison by selecting only those computed modes with oscillation energies typical of acoustic modes; for each harmonic degree the observed frequencies are generally matched with the closest model frequencies, although the assignment of mode order is occasionally shifted to ensure that the differences vary relatively smoothly 

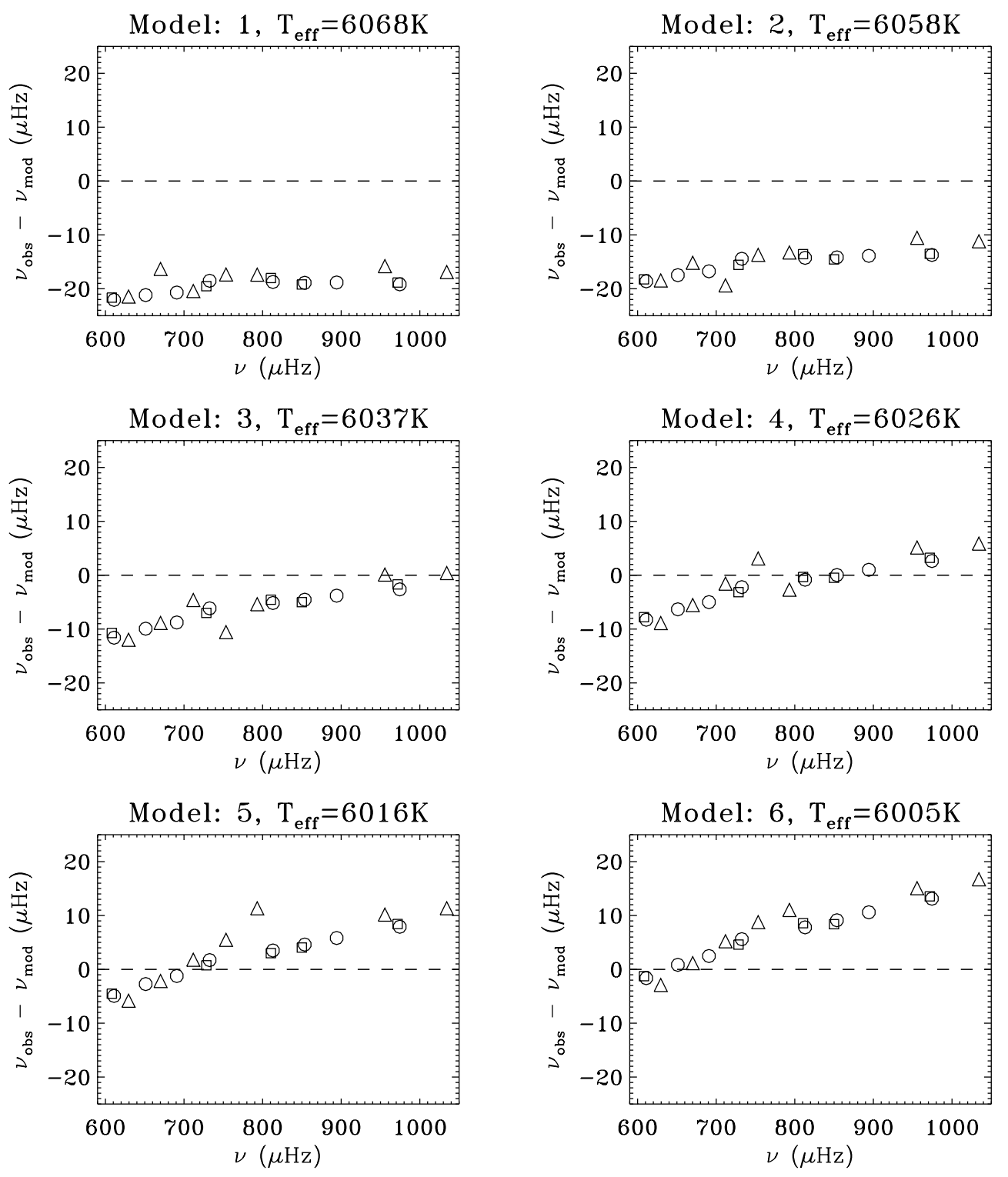

Fig. 11. Same as Fig. 10, but with overshooting $\left(\alpha_{\mathrm{ov}}=0.1\right)$ and with characteristics described in Table 2.

with frequency. Needless to say, care has been taken not to use the same theoretical mode twice. In the comparison a single observed mode, with $l=1$ and $v=749.3 \mu \mathrm{Hz}$, was excluded: as discussed in more detail below, and by Kjeldsen et al. (2003), this appears to be an observed case of an avoided crossing between two modes; since no similar pair can in general be expected in the computed spectrum (unless the model is very similar to the actual structure of the star) the presence of the pair in the observed set would cause an unreasonable shift in the mode assignment and hence in the frequency differences.

Figures 10-12 reflect the expected decrease in the computed frequencies as the models evolve and the effective temperature decreases. (The jump between Models 2 and 3 in Fig. 12 reflects a shift by one in the mode-order assignment of computed modes.) Also, the differences are quite small and pass close to zero, yielding models which satisfy the condition $v_{\mathrm{obs}} \simeq v_{\text {mod }}$; on this basis one might identify Model 3 of Table 1, Model 4 of Table 2 and Model 5 of Table 3 as the ones which best represent the structure of $\eta$ Boo, according to the observational information available. However, this comparison requires some care. It is well known in the solar case (e.g. Christensen-Dalsgaard 1996) that even for models that otherwise reflect the helioseismic structure of the Sun there typically remain substantial differences between the computed and observed frequencies. These are believed to be associated with inadequate modelling of the uppermost layers of the Sun, where nonadiabatic effects and effects of turbulent convection are typically neglected in the modelling. Effects arising from this layer are generally functions just of frequency for low-degree modes and are furthermore very small at low frequency where the inadequately treated layer is well above the upper turning points of the modes (e.g. Christensen-Dalsgaard $\&$ Thompson 1997). Due to their strong frequency dependence such effects also influence the large separation. Similar effects, although of uncertain magnitude, must be expected in the case of $\eta$ Boo (see also Christensen-Dalsgaard et al. 1995b). 

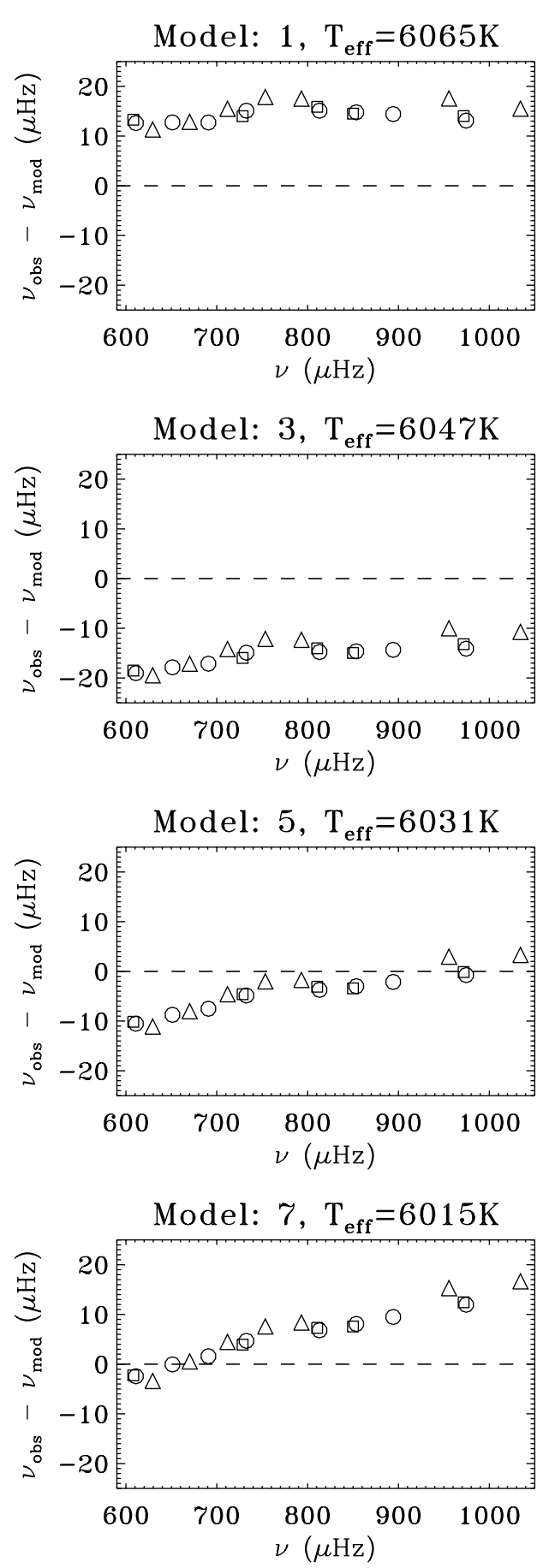
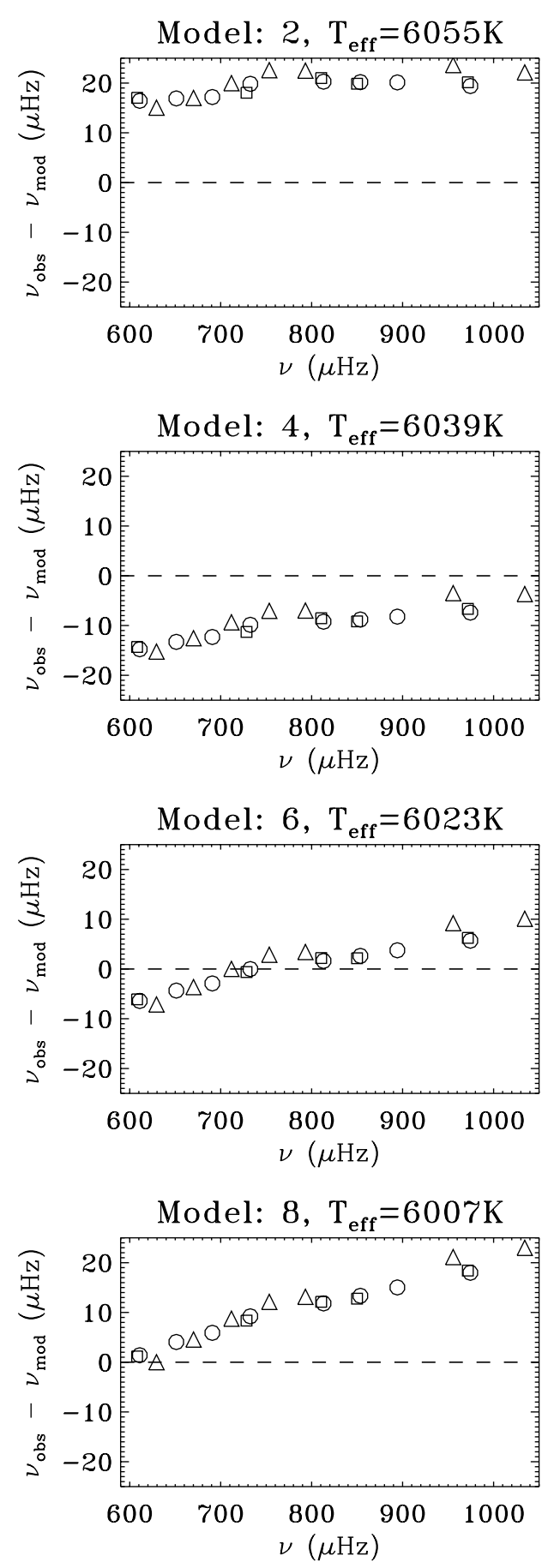

Fig. 12. Same as Fig. 10, but with overshooting $\left(\alpha_{\mathrm{ov}}=0.2\right)$ and with characteristics described in Table 3.

A closer inspection of Figs. 7-9 and Figs. 10-12 does indeed suggest problems of this nature. Consider the model without overshooting. According to Fig. 7 the best fit to the large separation, averaged over the central frequency region, occurs for Model 1; yet as is clear from Fig. 10 in this case the computed frequencies are substantially higher than the observed values. On the other hand, the agreement between the observed and computed large separations is reflected in the fact that the frequency differences are essentially independent of frequency. In Model 3, where the agreement between the frequencies is the closest, the differences show a definite slope corresponding to the fact that the computed large separation is smaller than the observed value. If one argues that the differences should be near zero at low frequency, where the nearsurface errors in the model have the smallest effect, one might choose instead Model 4 where, however, the slope in the differences is even more pronounced. A similar behaviour is found for the other two evolution sequences. It is evident that none of the models provides a perfect fit to the data.

In order to study the fine structure of the observed and computed oscillation frequency spectra, it is convenient to use an echelle diagram (Grec et al. 1983). Each frequency can be expressed in terms of an integer multiple of $\Delta v$, according to

$v_{n, l}=v_{0}+k \Delta v+\tilde{v}$ 


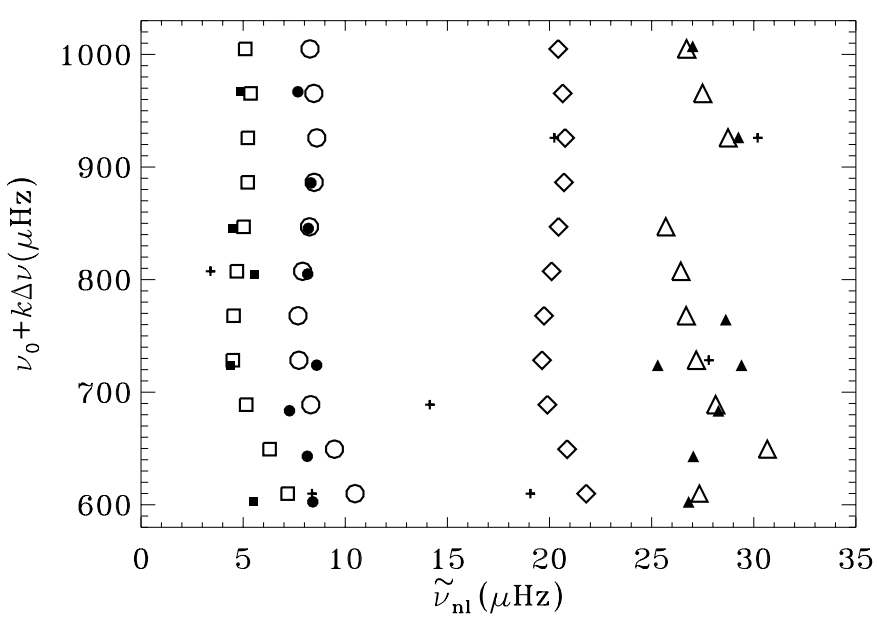

Fig. 13. Echelle diagram based on observed and computed frequencies. The filled symbols show frequencies observed by Kjeldsen et al. (2003) plotted with $\Delta v=40.47 \mu \mathrm{Hz}$ and a reference frequency $v_{0}=724.0 \mu \mathrm{Hz}$. The open symbols show computed frequencies for Model 3 in Table 1 which neglects overshooting, and were obtained with $\Delta v=39.5 \mu \mathrm{Hz}$ and $v_{0}=728.4 \mu \mathrm{Hz}$. Circles are used for modes with $l=0$, triangles for $l=1$, squares for $l=2$, diamonds for $l=3$. The size of the open symbols indicates the relative surface amplitude of oscillation of the modes. Crosses are employed for modes with small predicted amplitude (e.g. g modes). Here the theoretical $\delta v_{0}=3.2 \mu \mathrm{Hz}$.

where $v_{0}$ is an arbitrary reference frequency, $k$ is an integer and $\tilde{v}$ is a residual frequency which lies in the range $0-\Delta v$. In the echelle diagram $v_{0}+k \Delta v$ is plotted against $\tilde{v}$.

The echelle diagrams for the theoretical and observed frequencies are shown in Figs. 13-15, obtained respectively for Model 3 of Table 1, Model 4 of Table 2 and Model 5 of Table 3. Each echelle diagram has been obtained using a different value of $v_{0}$ respectively for the observed and theoretical frequencies, chosen to provide as close an overlap as possible between the two cases. To correct for the effects of near-surface errors, discussed above, different values of $\Delta v$ and $v_{0}$ have been used for each set of computed frequencies; for the observations, the average $\Delta v=40.47 \mu \mathrm{Hz}$, and the same value of $v_{0}=724 \mu \mathrm{Hz}$ was used in all cases.

The size of the symbols is proportional to the theoretical oscillation amplitudes of $\mathrm{p}$ modes, relative to the amplitudes of radial modes with the same frequency, computed by assuming all modes at a given frequency to have the same energy as explained in more detail by Christensen-Dalsgaard et al. (1995a); $\mathrm{g}$ modes and $\mathrm{p}$ modes with low surface amplitude are shown as small crosses.

Figure 13 is obtained for Model 3 of Table 1 which does not include overshooting and has a large frequency separation $\Delta v=39.5 \mu \mathrm{Hz}$. Here, the symbols for $l=0$ and $l=2$ run parallel, as predicted by Eq. (2), with a small frequency separation $\delta v_{0}=3.2 \mu \mathrm{Hz}$ which is close to the observed value of $3.06 \mu \mathrm{Hz}$. The comparison indicates that frequencies calculated for $l=0$ and $l=2$ are consistent with observations (filled symbols). The model frequencies for modes with $l=1$, as already explained, are strongly affected by avoided crossings and they deviate from the expected asymptotical behaviour.

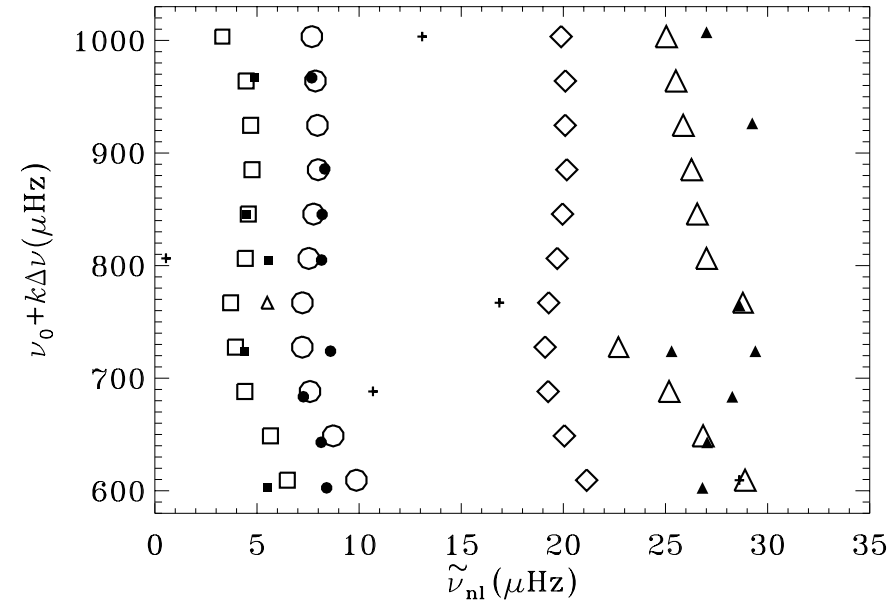

Fig. 14. Echelle diagram obtained for Model 4 of Table 2 that considers overshooting from the convective core with $\alpha_{\mathrm{ov}}=0.1$. The computed frequencies have been analyzed using $\Delta v=39.4 \mu \mathrm{Hz}$ and $v_{0}=727.6 \mu \mathrm{Hz}$. The observed frequencies have been plotted by employing $v_{0}=724.0 \mu \mathrm{Hz}$. Symbols have the same significance as in Fig. 13. Here the theoretical $\delta v_{0}=2.8 \mu \mathrm{Hz}$.

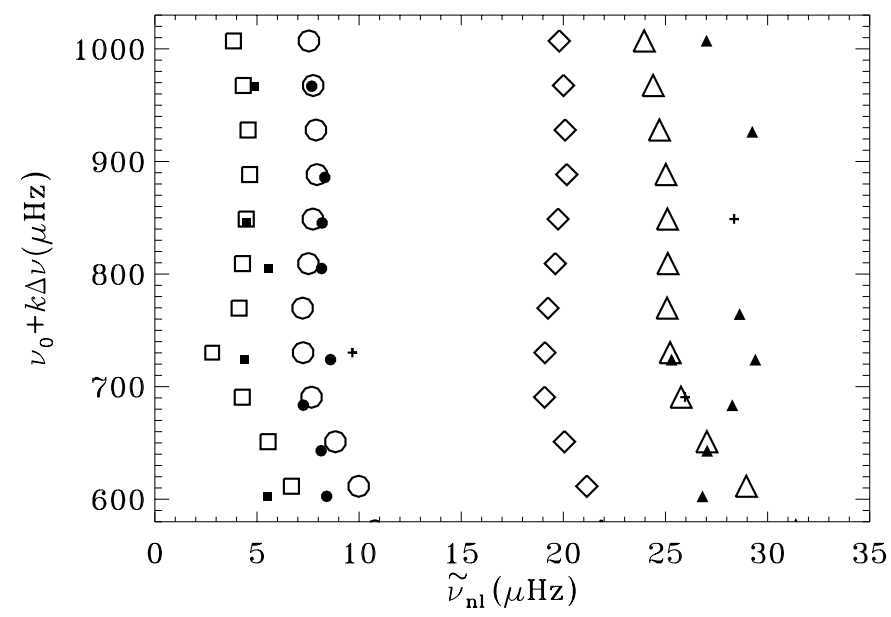

Fig. 15. Echelle diagram obtained for Model 5 of Table 3 that considers overshooting from the convective core with $\alpha_{\mathrm{ov}}=0.2$. Here, the computed frequencies have been analyzed with $\Delta v=39.6 \mu \mathrm{Hz}$, and $v_{0}=730.2 \mu \mathrm{Hz}$. The observed frequencies have been plotted by using $v_{0}=724.0 \mu \mathrm{Hz}$. Symbols have the same significance as in Fig. 13. The theoretical $\delta v_{0}=3.3 \mu \mathrm{Hz}$.

Interestingly, as already noted by Christensen-Dalsgaard et al. (1995a) and Guenther \& Demarque (1996), the observed points appear to show a qualitatively similar behaviour, although it differs in detail from the computed values; in particular, at $v_{0}+k \Delta v \simeq 725 \mu \mathrm{Hz}$ there is a closely spaced pair of observed modes with $l=1$, highly suggestive of an avoided crossing.

Figure 14 obtained for Model 4 of Table 2, which includes overshoot with $\alpha_{\mathrm{ov}}=0.1$, shows that frequencies of a model with a modest overshooting behave as those of a model which does not include overshooting, and modes with $l=1$ are still affected by avoided crossing, but only at lower frequencies. Model 4 of Table 2 is characterized by a large frequency separation $\Delta v=39.4 \mu \mathrm{Hz}$ and a small frequency separation $\delta v_{0}=2.8 \mu \mathrm{Hz}$. 
On the other hand, the echelle diagram in Fig. 15, obtained for the Model 5 of Table 3 with $\alpha_{\mathrm{ov}}=0.2$, shows that the computed frequencies for all the modes follow the prediction of Eq. (2) with no occurrence of resonance between p and $\mathrm{g}$ modes. For this model, we found that $\delta v_{0}=3.3 \mu \mathrm{Hz}$ and $\Delta v=39.6 \mu \mathrm{Hz}$. Both Figs. 14 and 15 show that there is, also in these cases, a generally reasonable agreement between theoretical values and observed data, although it seems that the model with $\alpha_{\mathrm{ov}}=0.1$, with some occurrences of avoided crossing, might represent a more appropriate choice.

\section{Conclusion}

Although the question of overshooting is quite open and can be settled only by the use of a more realistic theory of convection, the results reported here indicate that both the overshooting and the "classical" models can reproduce the observed parameters, such as effective temperature and luminosity of $\eta$ Boo. The location in the H-R diagram indicates that this star is in the postmain-sequence phase of evolution, with a mass in the range $M=1.64-1.75 M_{\odot}$. The latter has been obtained by assuming the most recent values of parallax, metallicity and effective temperature. We have also shown that models which fit the position of $\eta$ Boo in the $\mathrm{H}-\mathrm{R}$ diagram may have a maximum overshooting extent of $\ell_{\mathrm{ov}}=0.25 H_{\mathrm{p}}$.

We found, from calculation of the theoretical oscillation spectrum of $\eta$ Boo, that the large frequency separation of the low- $l \mathrm{p}$ modes is about $40 \mu \mathrm{Hz}$, while the small separation between modes with $l=0$ and $l=2$ is about $3 \mu \mathrm{Hz}$. Thus, models without and with convective-core overshooting can be constructed which are consistent with the observed frequency separations.

Our results are consistent with two main evolutionary scenarios for $\eta$ Boo, represented by Model 3 of Table 1 and Model 5 of Table 3: (i) a more evolved star without core overshooting and whose oscillation spectrum contains frequencies of nonradial modes with mixed character due to avoided crossings; (ii) a less evolved star which includes overshooting from the convective core and has p modes in the observed frequency range which show no mixed character and follow the asymptotic theory. Intermediate cases are also possible as represented by a model which includes modest convective overshooting from the core and with typical characteristics of both the two above mentioned scenarios (e.g., Model 4 of Table 2).

The quality of the existing frequency observations does not allow a clear distinction between the proposed scenarios; however, we note that in the echelle diagrams, Figs. 13-15, the observed $l=1$ modes show some tendency for deviation from the asymptotic behaviour; this is reproduced by the computed frequencies, at least qualitatively, in the models with no or modest overshooting (Figs. 13 and 14), but not in the model with more substantial overshooting (Fig. 15), possibly providing a hint that the former models might be preferred. Also, it is striking although hardly decisive that the evolution sequence with $\alpha_{\mathrm{ov}}=0.2$ places the star in a very rapid phase of evolution, which might be less likely to be observed. However, it should be noticed that none of the models provides a perfect fit to the observations; this is seen very clearly from the conflicting identifications obtained by comparing the large separations and the individual frequencies with the observations. These comparisons are undoubtedly affected by the errors in the treatment of the superficial layers in the model; however, based on past helioseismic experience it is also possible that improvements in the equation of state, inclusion of diffusion and settling or possibly a different hydrogen abundance, will have a significant effect. As better data become available, from additional coordinated ground-based observations and from space, these issues should be addressed in more comprehensive modelling and fits to the observed frequencies.

Most importantly, our results show that frequencies, measured with the accuracy which can be expected within the next few years by observing from space, will permit a discrimination among the different scenarios, clarifying the role of overshooting from convective cores in stellar evolution and likely elucidating other uncertain aspects of stellar modelling.

Acknowledgements. We thank the anonymous referee for perceptive comments which have led to substantial improvements to the text. This research was supported by the Danish National Research Foundation, through the establishment of the Theoretical Astrophysics Center.

\section{References}

Bahcall, J. N., \& Pinsonneault, M. H. 1995, Rev. Mod. Phys., 67, 781 Bedding, T. R., Kjeldsen, H., \& Christensen-Dalsgaard, J. 1997, in The 10th Cambridge Workshop on Cool Stars, Stellar Systems and the Sun, ed. R. A. Donahue, \& J. A. Bookbinder (San Francisco: ASP), ASP Conf. Ser., 154, 741

Bell, R. A., \& Gustafsson, B. 1989, MNRAS, 236, 653

Blackwell, D. E., \& Lynas-Gray, E. A. 1994, A\&A, 282, 899

Brown, T. M., Kennelly, E. J., Korzennik, S. G., et al. 1997, ApJ, 475, 322

Carrier, F., Bouchy, F., \& Eggenberger, P. 2003, in Asteroseismology across the H-R diagram, ed. M. J. Thompson, M. S. Cunha, \& M. J. P. F. G. Monteiro (Dordrecht: Kluwer Academic Publishers), in press

Christensen-Dalsgaard, J. 1982, MNRAS, 199, 735

Christensen-Dalsgaard, J. 1988, in Advances in Helio- and Asteroseismology, ed. J. Christensen-Dalsgaard, \& S. Frandsen (Dordrecht: Reidel), IAU Symp., 123, 295

Christensen-Dalsgaard, J. 1996, in The Structure of the Sun, ed. T. Roca Cortés, \& F. Sánchez (Cambridge: Cambridge University Press), 47

Christensen-Dalsgaard, J. 2002, in 1st Eddington Workshop on Stellar Structure and Habitable Planet Finding, ed. F. Favata, I. W. Roxburgh, \& D. Galadi (Noordwijk: ESA Publications Division), ESA SP-485, 25

Christensen-Dalsgaard, J., \& Berthomieu, G. 1991, in Solar Interior and Atmosphere, ed. A. N. Cox, W. C. Livingston, \& M. Matthews, Space Sci. Ser. (Tucson: Univ. of Arizona Press), 401

Christensen-Dalsgaard, J., \& Thompson, M. J. 1997, MNRAS, 284, 527

Christensen-Dalsgaard, J., Bedding, T. R., \& Kjeldsen, H. 1995a, ApJ, 443, L29

Christensen-Dalsgaard, J., Bedding, T. R., Houdek, G., et al. 1995b, in Astrophysical Applications of Stellar Pulsation, Proc. IAU Colloq. 155, ed. R. S. Stobie, \& P. A. Whitelock (San Francisco: ASP), ASP Conf. Ser., 83, 447

Eggleton, P. P., Faulkner, J., \& Flannery, B. P. 1973, A\&A, 23, 325 
Flower, P. J. 1996, ApJ, 469, 355

Grec, G., Fossat, E., \& Pomerantz, A. 1983, Sol. Phys., 82, 55

Guenther, D. B., \& Demarque, P. 1996, ApJ, 456, 798

Houdek, G., Balmforth, N. J., Christensen-Dalsgaard, J., \& Gough, D. O. 1999, A\&A, 351, 582

Iglesias, C. A., \& Rogers, F. J. 1996, ApJ, 464, 943

Kjeldsen, H., \& Bedding, T. R. 1995, A\&A, 293, 87

Kjeldsen, H., Bedding, T. R., Viskum, M., \& Frandsen, S. 1995, ApJ, 109,1313

Kjeldsen, H., Bedding, T. R., Baldry, I. K., et al. 2002, in Radial and Nonradial Pulsations as Probes of stellar Physics, Proc. IAU Colloq. 185, ed. C. Aerts, T. R. Bedding, \& J. ChristensenDalsgaard (San Francisco: ASP), ASP Conf. Ser., 259, 470
Kjeldsen, H., Bedding, T. R., Baldry, I. K., et al. 2003, AJ, in press Lèbre, A., de Laverny, P., de Medeiros, J. R., Charbonnel, C., \& da Silva, L. 1999, A\&A, 345, 936

Mallik, S. V. 1999, A\&A, 352, 495

Matthews, J. 1998, in Structure and Dynamics of the Interior of the Sun and Sun-like Stars, ed. S. G. Korzennik, \& A. Wilson (Noordwijk: ESA Publications Division), ESA SP-418, 395

Scuflaire, R. 1974, A\&A, 36, 107

Shaviv, G., \& Salpeter, E. E. 1973, ApJ, 184, 191

Taylor, B. J. 1996, ApJS, 102, 105

Tassoul, M. 1980, ApJS, 43, 469

Vandakurov, Yu V. 1967, AZh, 44, 786 (English trans.: SvA, 11, 630) 